\title{
Comparison between Average Charge States and Abundances of Ions in CMEs and the Slow Solar Wind
}

\author{
A. A. Reinard*, T. H. Zurbuchen*, L. A. Fisk*, S. T. Lepri*, R. M. Skoug ${ }^{\dagger}$ and G. \\ Gloeckler**
}

${ }^{*}$ Department of Atmospheric, Oceanic, and Space Science, University of Michigan, Ann Arbor, MI, 48109, USA

${ }^{\dagger}$ Space and Atmospheric Sciences Group, Los Alamos National Lab,Los Alamos, NM 87545

${ }^{* *}$ Department of Physics, University of Maryland, College Park, MD, 20742, Department of Atmospheric, Oceanic, and Space Science, University of Michigan, Ann Arbor, MI, 48109, USA

\begin{abstract}
.
We present results from a comparison of CME and slow solar wind ejecta detected at the ACE spacecraft in 1998 and 1999. CME events were identified based on the observation of counterstreaming halo electrons from SWEPAM data. We discuss the compositional signatures in the framework of a recent model of the coronal magnetic field by Fisk and Schwadron [1]. We conclude that slow solar wind and CMEs have a common source in the corona, presumably coronal loops. The largest amount of fractionation is found in helium and in charge state composition. The former is related to collisional effects in the corona and the latter is attributed to the anomalous heating and propagation properties of some CMEs.
\end{abstract}

\section{INTRODUCTION}

Coronal mass ejections (CMEs) are eruptions of coronal magnetic field and plasma into the solar wind. The first continuous space-borne observations of CME ejections were from coronagraphs on OSO-7 and Skylab in the early 1970 s $[2,3]$. The mechanisms responsible for these eruptions are not well understood [4]. CMEs in the vicinity of the Earth are identified based on a subset of specific characteristics including, but not limited to, the following: descending velocity profiles, enhanced and smoothly rotating magnetic field, low in situ kinetic temperatures, and enhanced $\alpha /$ proton ratios. In addition, the closed nature of the magnetic field lines of a CME allows identification based on counterstreaming halo electrons [5].

For this investigation we use data from the SWICS (Solar Wind Ion Composition Spectrometer), MAG (Magnetometer instrument) and SWEPAM (Solar Wind Electron, Proton, and Alpha Monitor) instruments on board the ACE (Advanced Composition Explorer) spacecraft. The SWICS instrument measures the composition of the solar wind by use of an electrostatic deflection system, a linear time of flight with post-acceleration, and a solid state detector. This process allows unambiguous determination of the mass and mass per charge of most ions. With this information we can distinguish different ion charge states and determine elemental abundances [6]. The MAG instrument consists of two magnetometers that measure the amplitude and direction of the interplanetary magnetic field [7]. The SWEPAM instrument consists of an ion spectrometer and an electron spectrometer which measure the solar wind proton, helium and electron distributions [8]. Our study utilizes counterstreaming halo electron events identified in the SWEPAM data to determine the periods of CME events.

Composition measurements provide a direct measure of the source conditions in the coronal environment. Compositional patterns observed in situ "freeze-in" during the expansion of the $\mathrm{CME}$ from the corona to $1 \mathrm{AU}$ and thus provides an important link between remote solar observations and in situ plasma observations. Composition signatures typically fall in two parts: ionic composition of given elements and elemental abundances.

Ionic compositional anomalies observed in some CMEs $[9,10,11]$ are caused by unusual thermal conditions of the CME plasma in the low corona or chromosphere. These signatures are typically found in $50 \%$ of all ejecta (identified by plasma properties) [12, 13]. The details of the freeze-in process are generally rather complex $[14,15]$ and strongly dependent on the electron temperature profile. Non-thermal properties of near solar electron distribution functions can also be reflected in the observed charge states $[16,17]$.

CP598, Solar and Galactic Composition, edited by R. F. Wimmer-Schweingruber (C) 2001 American Institute of Physics 0-7354-0042-3/01/\$18.00 
The elemental composition reflects the coronal source of the CME prior to the ejection into space. The slow solar wind and most CMEs observed in the ecliptic plane are fractionated relative to the photospheric composition. Typically, elements of low First Ionization Potential (FIP), such as $\mathrm{Fe}, \mathrm{Mg}, \mathrm{Si}$, are enhanced over high-FIP elements $(\mathrm{Ne}, \mathrm{He})$ [18]. This fractionation presumably occurs in closed magnetic structures $[19,20]$. This phenomenon has recently been observed using spectroscopic composition measurements on SOHO [21].

Using these composition parameters, we now address the following important questions:

1. Is there a difference between the source and expansion properties of slow wind and CME plasma?

2. What are the consequences of such differences in the appearance of CMEs at $1 \mathrm{AU}$ ?

These questions will be studied using the average properties of CMEs and slow solar wind periods. We will then discuss these data in the framework of a solar coronal model by Fisk and Schwadron [1].

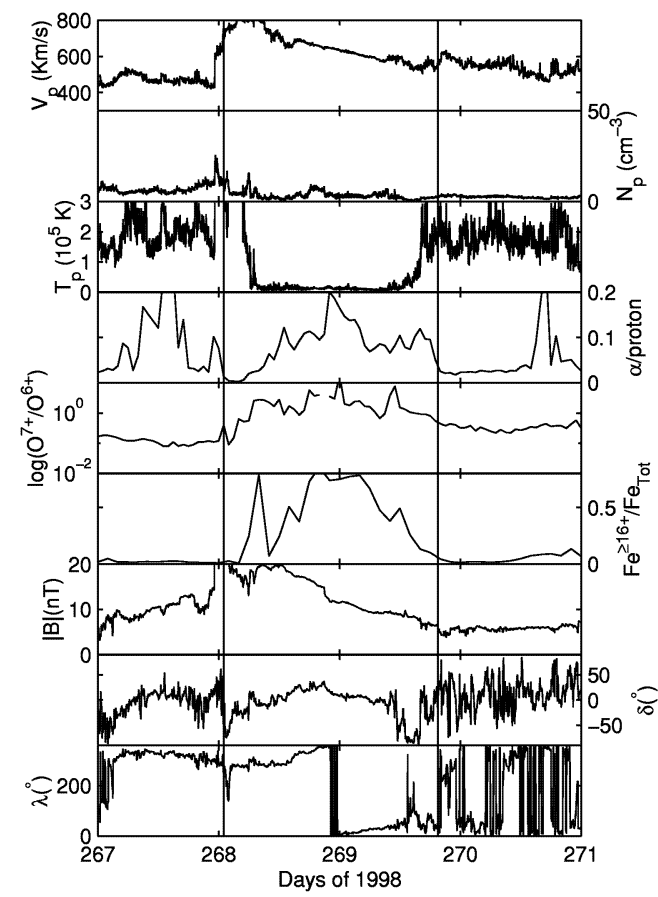

FIGURE 1. CME event identified by counterstreaming halo electrons (solid vertical lines). This event has increased charge states and $\alpha /$ proton ratio and decreased kinetic temperature.

\section{GENERAL CHARACTERISTICS OF CME COMPOSITION}

In this section we compare the compositional patterns of CMEs and slow solar wind. CMEs are identified based on the observation of counterstreaming halo electrons by the SWEPAM instrument. Figure 1 shows an example of one CME in this study. The period of counterstreaming electrons occurred between the dark vertical lines. The first panel shows the proton velocity $\left(V_{p}\right)$ which has a typical expansion profile consisting of a sharp increase followed by a slow decrease. The second and third panels show proton density $\left(N_{p}\right)$ and kinetic temperature $\left(T_{p}\right)$. The kinetic temperature clearly decreases during this CME event. The fourth through sixth panels show composition parameters: $\alpha$ /proton ratio, $\mathrm{O}^{+7} / \mathrm{O}^{+6}$ ratio, and the abundance of $\mathrm{Fe}^{\geq+16}$ divided by total iron abundance. These compositional parameters are enhanced during this CME. The seventh through ninth panels show total magnetic field magnitude and field components latitude $(\delta)$ and longitude $(\lambda)$.

Our study included a total of $56 \mathrm{CME}$ events during 1998 and early 1999. For each CME the composition parameters shown in Figure 1 (in addition to $\mathrm{He} / \mathrm{O}$ and $\mathrm{Fe} / \mathrm{O}$ ) were averaged over the entire $\mathrm{CME}$ as defined by the presence of counterstreaming electrons. This procedure allows us to do a statistical survey of CME composition. It should be emphasized that the data presented in this paper are, in some cases, averages over a long period of time and so effects such as high freeze-in temperatures and high $\mathrm{He} / \mathrm{H}$, among others, are muted if the enhancements only occurred over a fraction of the period of counterstreaming electrons.

For each CME time period we selected a "slow solar wind" time period by subtracting six days from the beginning time of the CME. This delay was corrected if there was any suspected interference with a previous CME or a high speed stream. The average speed of all "slow solar wind" periods is $415 \pm 100 \mathrm{~km} / \mathrm{s}$. Average values of several parameters were calculated for these slow solar wind periods over time intervals similar to the CME durations. We note that there are sometimes significant variations in the duration of CME associated times [22], which may indicate that an ejecta observed at 1 AU may be composed of a number of individual CMEs.

We now present survey plots that compare average CME composition with slow solar wind composition. The $\mathrm{O}^{+7} / \mathrm{O}^{+6}$ ratio is used to order these data. When possible, we include average coronal hole composition from Ulysses during the south coronal hole passage between September 8-18, 1994. Each of the survey plots has the same format as described in Figure 2.

We first show a comparison of $\mathrm{O}^{+7} / \mathrm{O}^{+6}$ and average iron charge states (Figure 2). Both quantities can be directly related to the electron temperature at their respective freeze-in points. $\mathrm{O}^{+7} / \mathrm{O}^{+6}$ typically freezes-in close to the solar surface $\left(<2 \mathrm{R}_{\odot}\right)$ and iron freezes-in further out (3-4 $\left.\mathrm{R}_{\odot}\right)$. The different freeze-in radii are a consequence of the different recombination rates of the two elements ([18] and references therein). Assuming a stan- 


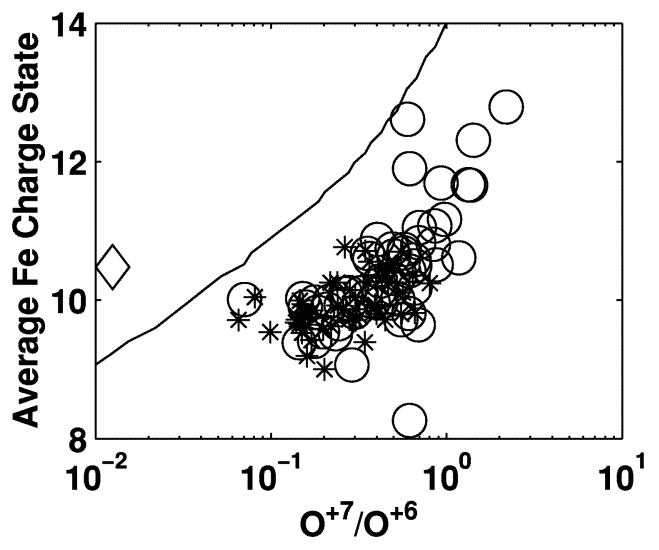

FIGURE 2. $\mathrm{O}^{+7} / \mathrm{O}^{+6}$ ratio versus the average iron charge state for CMEs identified by counterstreaming halo electrons (circles), slow solar wind periods (stars) in 1998 and 1999, and an average coronal hole value (diamond). The line indicates values of collisional equilibrium of iron and oxygen with thermal electrons. $\mathrm{O}^{+7} / \mathrm{O}^{+6}$ shows a positive correlation with iron charge state in both the slow solar wind and CME periods.

dard electron temperature profile [23] in which a temperature peak occurs around $2 \mathrm{R}_{\odot}$, oxygen freezes in at a positive temperature gradient and iron freezes in at a negative temperature gradient. Small changes in velocity, temperature, or density would thus tend to cause an anti-correlation in oxygen and iron freeze-in temperatures [32]. Figure 2 clearly shows a positive correlation between $\mathrm{O}^{+7} / \mathrm{O}^{+6}$ and the average iron charge state in both the slow solar wind and CME associated plasma. Note also that only CMEs are associated with the hottest freeze-in temperatures $[9,10,24]$. For comparison, local thermodynamic equilibrium values $[25,26]$ have been plotted in a solid line. All CME and slow solar wind points lie below this line, indicating a lower freeze-in temperature for iron than oxygen, which is consistent with the effects of a negative temperature gradient in the corona and contrary to the standard electron temperature profile.

Figure 3 compares the ion kinetic temperature with $\mathrm{O}^{+7} / \mathrm{O}^{+6}$. We use helium rather than electron kinetic temperature due to the smaller dependence on spacecraft charging effects. The anti-correlation between $\mathrm{O}^{+7} / \mathrm{O}^{+6}$ and the kinetic temperature may be a signature of thermally-driven expansion close to the Sun. Note that some CMEs have a relatively hot in situ kinetic temperature, in contrast with the typical picture of a CME event. These events bear closer investigation. Figure 4 shows an equivalent plot for the average speed. Helium velocity is used because it is a very good approximation for velocities of the heavier elements and because statistics allow a more accurate measurement. Typically, CME speeds exceed that of slow solar wind events and show

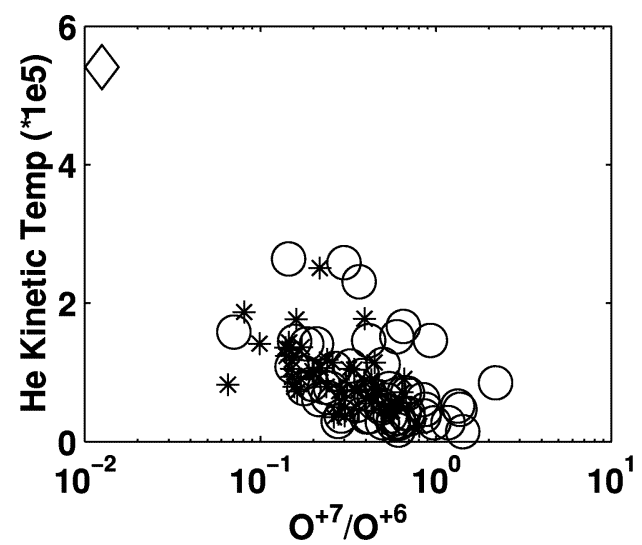

FIGURE 3. Same as Figure 2 but for $\mathrm{O}^{+7} / \mathrm{O}^{+6}$ versus in situ helium kinetic temperature. $\mathrm{O}^{+7} / \mathrm{O}^{+6}$ and kinetic temperature are inversely related.

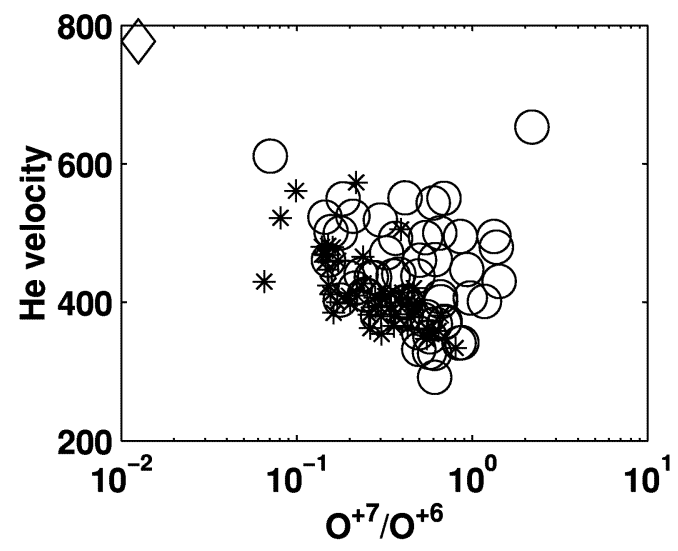

FIGURE 4. Same as Figure 2 but for $\mathrm{O}^{+7} / \mathrm{O}^{+6}$ versus in situ helium velocity. $\mathrm{O}^{+7} / \mathrm{O}^{+6}$ and velocity are inversely correlated for slow solar wind, while CME events have a broader range in $\mathrm{O}^{+7} / \mathrm{O}^{+6}$ for a given velocity. The average $\mathrm{CME}$ speed is clearly larger than average slow solar wind speed.

a larger variability. There is a possible anti-correlation between $\mathrm{O}^{+7} / \mathrm{O}^{+6}$ and speed in CME events, while for the slow solar wind the anti-correlation is clear.

In Figure 5 we see no apparent correlation between $\mathrm{O}^{+7} / \mathrm{O}^{+6}$ and $\mathrm{Fe} / \mathrm{O}$. Fe/O is generally $\sim 0.1$ for FIP associated plasma and $\sim 0.04$ in the photosphere [27]. Our values in both CME and slow solar wind events fall between those values. CME events, once again, greatly overlap the slow solar wind events, and account for the hotter events and those events with the highest Fe/O. This observation is probably due to $\mathrm{CME}$ events being a more extreme examples of slow wind phenomenon.

$\mathrm{He} / \mathrm{O}$ and $\mathrm{He} / \mathrm{H}$ give another measure of fractionation as helium is a very-high FIP element. Our results (Figures 6 and 7) indicate that there is no ordering due to frac- 


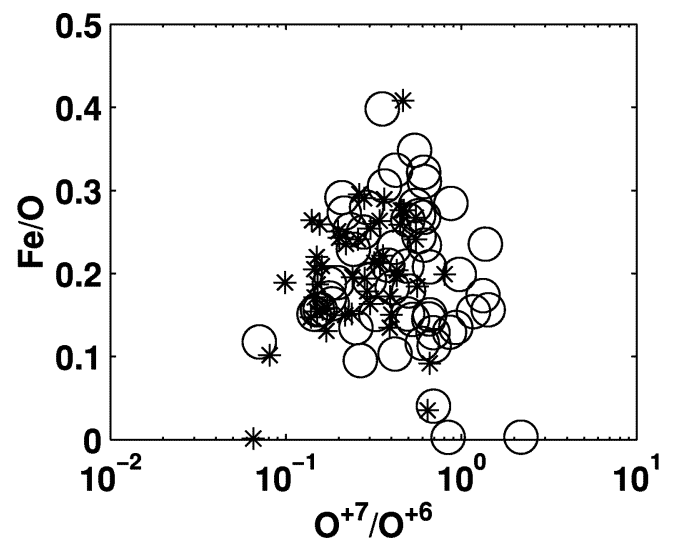

FIGURE 5. Same as Figure 2 but for $\mathrm{O}^{+7} / \mathrm{O}^{+6}$ versus the iron to oxygen ratio. No relation is apparent.

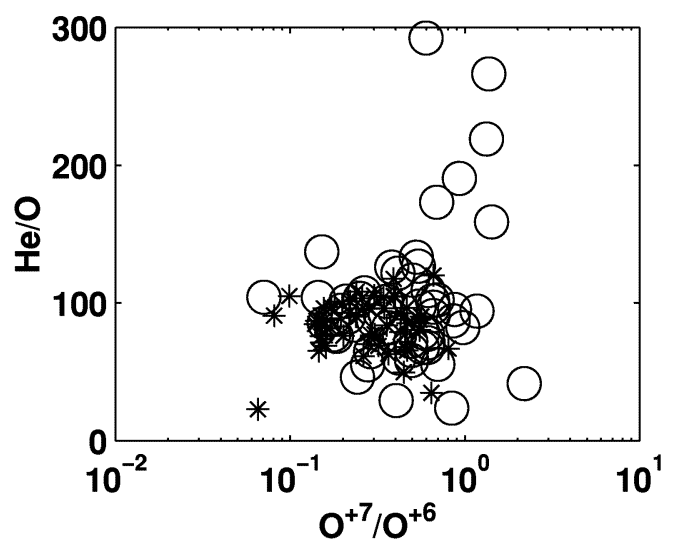

FIGURE 6. Same as Figure 2 but for $\mathrm{O}^{+7} / \mathrm{O}^{+6}$ versus the helium to oxygen ratio. CMEs with high values of $\mathrm{O}^{+7} / \mathrm{O}^{+6}$ can show enhanced $\mathrm{He} / \mathrm{O}$.

tionation based on $\mathrm{He} / \mathrm{O}$ or $\mathrm{He} / \mathrm{H}$ except in very hot $\mathrm{CME}$ events, where a large amount of variability occurs. This variability is much larger than in any other ionic species studied here. $\mathrm{He}^{2+}$ has the smallest collisional crosssection of all solar wind ions [28] and is therefore less coupled to the solar wind protons than any other heavy ion species. The consequent fractionation may therefore be of different nature than the FIP fractionation process, as indicated by comparisons of Figures 5 and 6 .

\section{DISCUSSION}

A recent series of papers by Fisk et al. [1, 29, 30] have argued that there is large-scale and relatively rapid transport of open magnetic flux in the solar corona. Transport occurs by a diffusive process in which open field lines reconnect with closed magnetic loops and execute a series

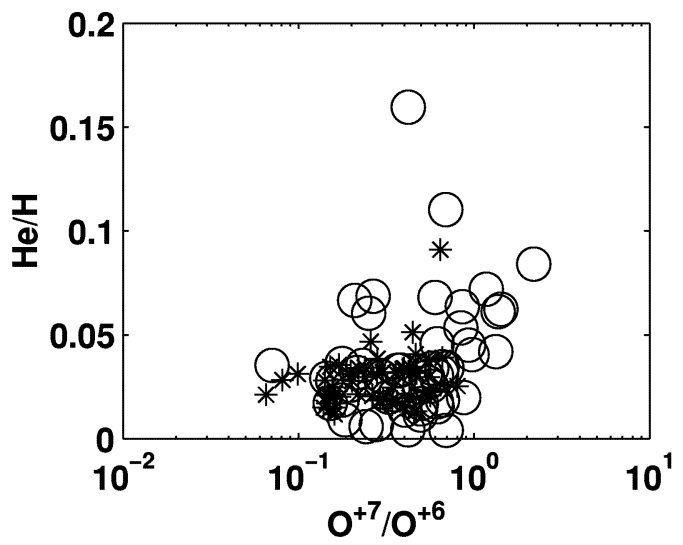

FIGURE 7. Same as Figure 2 but for $\mathrm{O}^{+7} / \mathrm{O}^{+6}$ versus the helium to hydrogen ratio. CMEs with high values of $\mathrm{O}^{+7} / \mathrm{O}^{+6}$ can have very high $\mathrm{He} / \mathrm{H}$

of random jumps in location. This process provides an explanation for the origin of the slow solar wind. The reconnection process and subsequent displacements of open field lines release energy into the solar corona that is sufficient to accelerate the solar wind [1,30]. Material stored on the closed field lines is also released to form the mass flux in the solar wind.

The closed magnetic loops referred to in this theory have been identified by Feldman et al. [21]. The loops have heights of order $100,000 \mathrm{~km}$, and electron freezein temperatures $\sim 1.4 \times 10^{6} \mathrm{~K}$. Of more significance, they are FIP-enhanced in their composition [31], strongly suggesting that they are the origin of the slow solar wind.

CMEs also originate in coronal loops. It is reasonable to expect, then, that the characteristics of the slow solar wind and CMEs should be similar, with perhaps CMEs being simply a more extreme version, i.e. originating from larger and hotter loops [22]. This expectation is consistent with the observations presented here, though a more in-depth study is needed to confirm this hypothesis.

\section{Low latitude corona has monotonically decreasing temperature}

In Figure 2 we see that in both the slow solar wind and in most CME events the average iron charge state varies between 9 and 11 and the $\mathrm{O}^{+7} / \mathrm{O}^{+6}$ ratio varies between 0.1 and 1 . Both data sets follow a similar trend which is linear. This result is inconsistent with both the slow solar wind and CME events arising from a corona with an electron temperature peak. If the peaked profile were valid in these periods we would expect oxygen and iron based electron temperatures to be anti-correlated as described in Ko et al., [32]. These results provide 
evidence that the slow solar wind and CME events both originate in a corona with a monotonically decreasing electron temperature profile.

The electron temperature of solar loops, $\sim 1.4 \times 10^{6} \mathrm{~K}$ [21], are consistent with charge states for $\mathrm{O}^{+7} / \mathrm{O}^{+6}$ that are observed in the heliosphere, i.e. the data are consistent with the freeze-in of $\mathrm{O}^{+7}$ and $\mathrm{O}^{+6}$ occurring in the loop. As the material expands out from the Sun after reconnection releases it from the loop, any subsequent heating is insufficient to overcome a temperature that we find decreases monotonically with distance. This expansion of the slow solar wind should be contrasted with the fast solar wind from the polar coronal hole, which Geiss et al. [18] determined had a temperature maximum that lies between the freeze-in points of $\mathrm{O}^{+7} / \mathrm{O}^{+6}$ and iron.

\section{CME expansion dominates the in situ kinetic temperatures}

Figures 3 and 4 show a decrease in kinetic temperature and velocity with increased $\mathrm{O}^{+7} / \mathrm{O}^{+6}$. This finding is a signature of expansion and subsequent cooling of the solar wind. The expansion process is driven by the internal pressure of the CME. We can indirectly measure the internal pressure through the $\mathrm{O}^{+7} / \mathrm{O}^{+6}$ ratio. Unless there is a fortuitous drop in the magnetic field or density, a sharp increase in electron temperature is indicative of a stronger internal CME pressure. The larger the initial pressure the faster the expansion in the low corona. This expansion results in adiabatic cooling of the plasma.

\section{Elemental composition of CMEs and slow solar wind is similar, but not well ordered by coronal freeze-in temperature}

Figure 5 indicates that CMEs and the slow solar wind have a similar elemental composition. However, we see no relationship between the $\mathrm{Fe} / \mathrm{O}$ and $\mathrm{O}^{+7} / \mathrm{O}^{+6}$ ratios, indicating that compositional parameters are not wellordered by the coronal temperature in the structures, presumably loops, that gave rise to the CMEs and the slow solar wind. To some extent this result is surprising. The mechanisms by which the low FIP elements are enhanced in loops, described by Schwadron et al. [20], involve wave heating on the loops. Such heating will take time, and should also increase the electron freeze-in temperature in the loops and thus the $\mathrm{O}^{+7} / \mathrm{O}^{+6}$ ratio. We might have expected, therefore, a more direct correlation between the $\mathrm{Fe} / \mathrm{O}$ and the $\mathrm{O}^{+7} / \mathrm{O}^{+6}$ ratios. We note that average solar wind measurements from Aellig et al. and references therein [35] showed an increase of $\log (\mathrm{Fe} / \mathrm{O})$ with $\log \left(\mathrm{O}^{+7} / \mathrm{O}^{+6}\right)$ during a study of 80 days in 1996 .
The differing results may be related to the observations occurring at different points in the solar cycle.

\section{Helium is enhanced in "hot" CME events}

Figures 6 and 7 show that in CMEs with very large $\mathrm{O}^{+7} / \mathrm{O}^{+6}$ helium can be enhanced (averages values for $\mathrm{He} / \mathrm{O}$ are $\sim 75$ in the slow solar wind and $\sim 115$ in the photosphere [27]). The primordial helium abundance relative to hydrogen is $\sim 8 \%$ (see [34] and references therein). In comparison, the average solar wind helium abundance is only $\sim 4 \%$. The depletion of helium presumably occurs in the chromosphere or the low corona [33]. The details of the fractionation process are currently not well understood. It was first suggested by Geiss et al. [28], that the fractionation may be related to insufficient Coulomb drag in the low corona that would deplete the corona of helium. If this is the case some energetic CMEs might pick up these low coronal helium enhancements and move them into the solar wind. Our results support this possibility.

\section{CONCLUSIONS}

We have presented a series of observations of the elemental and charge-state composition in CMEs and the slow solar wind, and of correlations between the composition. The most striking conclusion are the similarities between the CMEs and the slow wind. They share a common elemental composition, monotonically decreasing kinetic temperature profiles, and an apparently similar expansion history. These observations are consistent with CMEs and the slow solar wind having a common origin of closed magnetic loops in the corona, with perhaps the only difference being the mechanism by which the material is released from the loops. In Fisk and Schwadron [1] a mechanism is provided for the release of material from loops to form the slow solar wind as open field lines reconnect with the closed loops. In CMEs the loops themselves are released into the solar wind.

\section{ACKNOWLEDGMENTS}

This work was supported in part by NASA grant S02GSRP-121 and by NASA contracts NAG5-2810 and NAG5-711. Work at Los Alamos was performed under the auspices of the US Department of Energy with financial support from the NASA ACE program. 


\section{REFERENCES}

1. Fisk, L. A., N. A. Schwadron, The behavior of the open magnetic flux of the sun, Astrophys. J., in press.

2. Tousey, R., The solar corona, Space Res., 13, 713, 1973.

3. Gosling, J. T., E. Hildner, R. M. MacQueen, R. H. Munro, A. I. Poland, and C. L. Ross, Mass ejections from the sun: a view from skylab, J. Geophys. Res., 79, 4581, 1974.

4. Klimchuk, J. A., Theory of coronal mass ejections, $J$. Geophys. Res., in press.

5. Gosling, J.T., Coronal mass ejections and magnetic flux ropes in interplanetary space, in Physics of Magnetic Flux Ropes, Washington D. C., 343, 1990.

6. Gloeckler, G, J Cain, F. M. Ipavich, E. O. Tums, P. Bedini, L. A. Fisk, T. H. Zurbuchen, P. Bochsler, J. Fischer, R. F. Wimmer-schweingruber, J. Geiss, and R. Kallenbach, Investigation of the composition of solar and interstellar matter using solar wind and pickup ion measurements with SWICS and SWIMS on the ACE spacecraft, Space Sci. Rev., 86, 497, 1998.

7. Smith, C. W., J. L'Heureux, N. Ness, F., M. H. Acuna, L. F. Burlaga, and J. Scheifele, The Ace magnetic fields experiment, Space Sci.Rev., 86, 613, 1998.

8. McComas, D. J., S. J. Bame, P. Barker, W. C. Feldman, J. L. Phillips, P. Riley, J. W. Griffee, Solar Wind Electron Proton Alpha Monitor (SWEPAM) for the Advanced Composition Explorer, Space Sci. Rev., 86, 563, 1998.

9. Fenimore, E. E., Solar wind flows associated with hot heavy ions, Astrophys. J., 235, 245, 1980.

10. Henke, T., J. Woch, U. Mall, S. Livi, B. Wilken, R. Schwenn, G. Gloecker, R. von Steiger, R. J. Forsyth, and A. Balogh, Differences in the $\mathrm{O}^{+7} / \mathrm{O}^{+6}$ ratio in magnetic cloud and non-cloud coronal mass ejections, Geophys. Res. Lett., 25, 3465, 1998.

11. Gloeckler, G., L. A. Fisk, S. Hefti, N. A. Schwadron, T. H. Zurbuchen, F. M. Ipavich, J. Geiss, P. Bochsler, R. F. Wimmer-Schweingruber, Unusual composition of the solar wind in the 2-3 May 1998 CME observed with SWICS on ACE, Geophys. Res. Lett., 26, 157, 1999.

12. Burlaga, L. F., R. M. Skoug, C. W. Smith, D. Webb, T. H. Zurbuchen, A. A. Reinard, Fast Ejecta during Ascending Phase of Solar Cycle 23: ACE observations, 1998-1999, J. Geophys. Res., in press.

13. Lepri, S. T., T. H. Zurbuchen, L. A. Fisk, I. G. Richardson, H. V. Cane, G. Gloeckler, Iron charge distribution as an identifier of interplanetary coronal mass ejections, $J$. Geophys. Res., in press.

14. Buergi, A. and J. Geiss, Helium and minor ions in the corona and solar wind: Dynamics and charge states, Sol. Phys., 103, 347, 1986.

15. Ko, Y-K, G. Gloeckler, C. Cohen, A. B. Galvin, Solar wind ionic charge states during the Ulysses pole-to-pole pass, J. Geophys. Res., 104, 17005, 1999.

16. Burgi, A., Effects of non-Maxwellian electron velocity distribution functions and nonspherical geometry on minor ions in the solar wind, J. Geophys. Res., 92, 1057, 1987.

17. Owocki, S. P., A. J. Hundhausen, The effect of a coronal shock wave on the solar wind ionization state, Astrophys. J., 274, 414, 1983.

18. Geiss, J., G. Gloeckler, R. von-Steiger, H. Balsiger, L. A. Fisk, A. B. Galvin, F. M. Ipavich., S. Livi., J. F. McKenzie, K. W. Ogilvie, B. Wilken, The southern high-speed stream: results from the SWICS instrument on Ulysses, Science, 268, 1033, 1995.

19. Zurbuchen, T. H., L. A. Fisk, G. Gloeckler, and N. A. Schwadron, Elemental and isotopic fractionation in closed magnetic structures, Space Sci. Rev., 85, 397, 1998.

20. Schwadron, N. A., L. A. Fisk, and T. H. Zurbuchen, Elemental fractionation in the slow solar wind, Astrophys. J., 521, 859, 1999.

21. Feldman, U., K. G. Widing, and H. P. Warren, Morphology of the quiet solar upper atmosphere in the $4 \mathrm{E}^{4}<\mathrm{T}_{e}<1.4 \mathrm{E}^{6} \mathrm{~K}$ temperature regime, Astrophys. J., 522, 1133, 1999.

22. Zurbuchen, T. H., S. Hefti, L. A. Fisk, G. Gloeckler, and N. A. Schwadron, Magnetic structure of the slow solar wind: Constraints from composition data, J. Geophys. Res., 105, 18327, 2000.

23. Vernazza, J. E., E. H. Avrett, R. Loeser, Structure of the solar chromosphere. III - Models of the EUV brightness components of the quiet-sun, Astrophys. J. Supplement Series, 45, 635, 1981.

24. Galvin, A. B., F. M. Ipavich, G. Gloeckler, D. Hovestadt, S. J. Bame, B. Klecker, M. Scholer, and B. T. Tsurutani, Solar wind iron charge states preceding a driver plasma, J. Geophys. Res., 92, 12069, 1987.

25. Arnaud,M, R. Rothenflug, An updated evaluation of recombination and ionization rates, Astronomy and Astrophysics Supplement Series, 60, 425, 1985.

26. Arnaud, M, J. Raymond, Iron ionization and recombination rates and ionization equilibrium, Astrophys. J., 398, 394, 1992.

27. von Steiger, R., J. Geiss, G. Gloeckler, Composition of the solar wind, in Cosmic Winds and the Heliosphere, University of Arizona Press, 581, 1997.

28. Geiss, J., P. Hirt, H. Leutwyler, On the acceleration and motion of ions in the corona and solar wind, Solar Phys., 12, 458, 1970.

29. Fisk, L. A., Motion of the footpoints of heliospheric magnetic field lines at the Sun: Implications for recurrent energetic particle events at high heliographic latitudes, $J$. Geophys. Res., 101, 15547, 1996.

30. Fisk, L. A., T. H. Zurbuchen, N. A. Schwadron, On the coronal magnetic field: consequences of large-scale motion, Astrophys. J., 521, 868, 1999.

31. Feldman, U., U. Schühle, K. G., Widing, J. M. Laming, Coronal composition above the solar equator and the north pole as determined from spectra acquired by the SUMER instrument on SOHO, Astrophys. J., 505, 999, 1998.

32. Ko, Y-K., L. A. Fisk, J. Geiss, G. Gloeckler,and M. Guhathakurta, An empirical study of the electron temperature and heavy ion velocities in the south polar coronal hole, Solar Phys., 171, 345, 1997.

33. Laming, J. M. and U. Feldman, The solar helium abundance in the outer corona determined from observations with SUMER/SOHO, Astrophys. J., 546, 552, 2001.

34. Coplan, M. A., K. W. Ogilvie, P. Bochsler, J. Geiss, Interpretation of He-3 abundance variations in the solar wind, Solar Phys., 93, 415, 1984.

35. Aellig, M. R., S. Hefti, H. Grünwaldt, P. Bochsler, P. Wurz, F. M. Ipavich, D. Hovestadt, The Fe/O elemental abundance ratio in the solar wind as observed with SOHO CELIAS CTOF, J. Geophys. Res., 104, 24769, 1999. 